\title{
Oscillateur microonde à diode Gunn de haute pureté spectrale stabilisé en fréquence par une cavité supraconductrice et électroniquement accordable
}

\author{
A. Kayyali, Pham Tu Manh, Nguyen Tuong Viet et L. Wartski \\ Institut d'Electronique Fondamentale (*), Bâtiment 220, Université Paris-XI, 91405 Orsay Cedex, France
}

(Reçu le 16 mars 1979, révisé le 29 juin 1979, accepté le 4 juillet 1979)

\begin{abstract}
Résumé. - L'utilisation comme élément de référence de fréquence, d'une cavité supraconductrice en niobium de coefficient de surtension élevé $\left(Q \simeq 10^{9}\right)$ et fonctionnant en transmission sur le mode $\mathrm{TM}_{010}$, a permis de réaliser, au moyen d'un montage de Pound modifié, un oscillateur à diode Gunn de haute pureté spectrale en bande X. La réduction du bruit de phase aux fréquences de Fourier élevées, grâce au filtrage du signal par la cavité stabilisatrice elle-même, a été observée : le taux de décroissance de la densité spectrale des fluctuations de phase est d'environ $20 \mathrm{~dB} /$ décade. Une variance $\sigma_{\mathrm{y}}$ des fluctuations de fréquence de $3 \times 10^{-13}$ a été mesurée sur une durée d'une seconde.

On montre qu'il est possible d'obtenir un accord électronique de fréquence de l'oscillateur stabilisé en effectuant une modulation de phase ou d'amplitude de l'onde à une fréquence intermédiaire $F(\simeq 1 \mathrm{MHz})$ au moyen d'un synthétiseur de fréquence. L'oscillateur ést alors asservi soit à la fréquence de résonance $f_{0}$ de la cavité, soit à une fréquence $f_{0} \pm F$. Dans ce dernier cas, une variation de $F$ entraîne la variation de la fréquence de l'oscillateur stabilisé.
\end{abstract}

\begin{abstract}
Using a high $Q\left(\simeq 10^{9}\right)$ niobium $\mathrm{TM}_{010}$ two port superconducting cavity as a frequency determining element, a X-band Gunn effect diode oscillator of high spectral purity has been realized by means of a modified Pound technique.

Phase noise reduction at high Fourier frequencies due to the filtering properties of the stabilizing cavity itself was observed : the rate of noise reduction is approximately $20 \mathrm{~dB} /$ decade for the spectral density of phase fluctuations. Fractional frequency fluctuations of $\sigma_{y} \simeq 3 \times 10^{-13}$ for a second averaging time has been measured.

It is shown that electronic frequency tuning of the stabilized oscillator can be achieved when realizing either a phase or amplitude modulation at an intermediate frequency $F(\simeq 1 \mathrm{MHz})$ by means of a frequency synthesizer. The oscillator is then locked either at the resonant frequency $f_{0}$ of the cavity or at a frequency $f_{0} \pm F$. In this latter case, variation of $F$ provides the frequency tuning of the stabilized oscillator.
\end{abstract}

Introduction. - Les progrès réalisés dans la technologie des cavités résonnantes supraconductrices ont permis d'obtenir des coefficients de surtension très élevés $\left(Q_{0} \simeq 10^{9}\right)$ ce qui permet leur utilisation comme éléments de référence dans les oscillateurs micro-ondes de grande pureté spectrale. Nous avons construit un tel oscillateur à diode Gunn en ondes centimétriques destiné au Laboratoire Primaire du Temps et des Fréquences en vue de la multiplication de fréquence dans l'IR lointain dans le cadre de la métrologie des fréquences des lasers moléculaires $\left({ }^{1}\right)$. La stabilisation de cet oscillateur est effectuée au

(*) Laboratoire associé au C.N.R.S.

( ${ }^{1}$ ) Ce travail a été réalisé dans le cadre d'un contrat avec le Bureau National de Métrologie. moyen d'un discriminateur de fréquence, d'autant plus sensible que le coefficient de surtension de la cavité est élevé. Le dispositif de stabilisation permet en outre de faire varier la valeur de la fréquence du signal stabilisé délivré par l'oscillateur.

1. Les cavités supraconductrices : leur construction et leur utilisation comme éléments de référence de fréquence. - Nous avons réalisé trois cavités cylindriques circulaires fonctionnant sur le mode $\mathrm{TM}_{010}$ et dont les fréquences de résonance se situent aux environs de $10 \mathrm{GHz}$. Chaque cavité est usinée en un seul bloc à partir de niobium massif, ce qui présente l'avantage, par rapport à la technique utilisée antérieurement, de supprimer le délicat problème des soudures par bombardement électronique 
de deux demi-cavités. Pour obtenir une rigidité mécanique suffisante et réduire ainsi l'influence des vibrations mécaniques, de la pression de radiation [1] et de la pression extérieure, il est nécessaire de conserver une épaisseur importante des parois, de l'ordre du rayon intérieur. Le traitement de surface consiste en un recuit à $1800^{\circ} \mathrm{C}$ dans un four à ultravide $\left(10^{-6} \mathrm{~Pa}\right)$, précédé d'un polissage chimique dans un bain composé de 60 volumes de $\mathrm{NO}_{3} \mathrm{H}$ concentré et de 40 volumes de $\mathrm{FH}$ à $40 \%$ à $0{ }^{\circ} \mathrm{C}$ [1].

Après traitement thermique, la cavité est assemblée à l'aide de joints d'indium à la fenêtre micro-onde étanche, puis pompée par un bâti ultravide $\left(10^{-7} \mathrm{~Pa}\right)$. Elle est ensuite scellée et placée dans une enceinte étanche qui l'isole des fluctuations de pression et de température du bain d'hélium dans le dispositif cryogénique. Un système de régulation électronique permet en outre de maintenir la température de la cavité utilisée comme élément de référence, constante à $10^{-4} \mathrm{~K}$ près au voisinage de $1,3 \mathrm{~K}$. A cette température, le coefficient de surtension à vide, pour chacune des cavités, est d'environ $10^{9}$. Chaque cavité est couplée par ses extrémités à un guide d'onde ou à un câble coaxial semi-rigide au moyen d'antennes axiales et peut ainsi fonctionner en transmission.

La fréquence de résonance d'une cavité supraconductrice dépend :

- De la géométrie et du mode de fonctionnement. Pour des expériences de synthèse de fréquence, la cavité doit avoir une fréquence de résonance bien précise $f_{0}$, avec une tolérance inférieure à $500 \mathrm{kHz}$. Cette précision sur la fréquence peut être obtenue par polissage chimique contrôlé de la surface intérieure de la cavité, à partir d'une cavité résonnant à une fréquence légèrement supérieure à $f_{0}$.

- De la température $T$ de fonctionnement; au voisinage de $T=1,6 \mathrm{~K}$, par exemple, on observe un glissement relatif de la fréquence d'environ $10^{-9} / \mathrm{K}$. Si l'on désire obtenir une stabilité de l'ordre de $10^{-14}$, une stabilisation de la température à $10^{-5} \mathrm{~K}$ près est nécessaire.

- De l'énergie emmagasinée dans la cavité. Lorsqu'on fait croître cette énergie, la fréquence de résonance diminue sous l'influence, d'une part de la pression de radiation sur les parois, d'autre part de la non-linéarité de la réactance de surface [2]. Nous avons mesuré une variation relative linéaire de fréquence de résonance de $10^{-6} / \mathrm{J}$. Lorsqu'une telle cavité est utilisée pour asservir la fréquence d'un oscillateur, elle transforme par conséquent la modulation d'amplitude de l'oscillateur en modulation de fréquence. Si l'on se fixe une stabilité de fréquence de $10^{-13}$, la modulation relative d'amplitude doit être inférieure à $10^{-4}$ pour une énergie injectée de l'ordre du millijoule.

2. Principe du dispositif électronique de stabilisation de fréquence. - 2.1 LE DISCRIMINATEUR DE
Pound MODIFIÉ. - L'oscillateur se trouvant à la température ambiante, l'utilisation comme élément de référence d'une cavité supraconductrice plongée dans un bain d'hélium (à une température comprise entre $1,3 \mathrm{~K}$ et $4,2 \mathrm{~K}$ ) nécessite l'emploi de lignes de transmission relativement longues soumises à de forts gradients de température. C'est pour s'affranchir des fluctuations de phases correspondant aux fluctuations de longueur de ligne que Trela et Fairbank [3] ont proposé une version modifiée du classique discriminateur de Pound à fréquence intermédiaire dont les avantages sont bien connus et dont nous rappelons sur la figure $1 a$ le principe de fonctionnement.

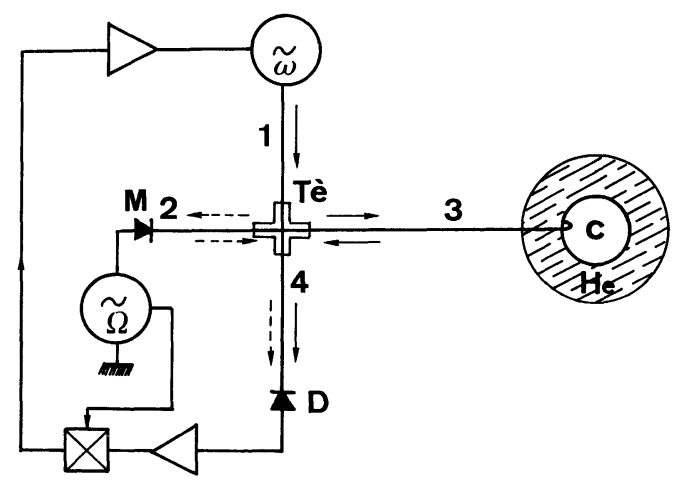

Fig. 1a. - Principe du discriminateur de Pound à fréquence intermédiaire.

[Block diagram of the IF Pound discriminator.]

L'onde de pulsation $\omega$ délivrée par l'oscillateur se partage, au niveau du té magique entre la voie (2) dans laquelle est placé le cristal modulateur adapté, et la voie (3) terminée par la cavité de référence. La voie (4) reçoit l'onde réfléchie par la cavité et les bandes latérales de modulation. Après détection quadratique et amplification du signal à la fréquence intermédiaire $\Omega$, la détection synchrone fournit un signal continu proportionnel à la partie imaginaire du coefficient de réflexion de la cavité (donc proportionnel à la fréquence au voisinage de la fréquence de résonance), à la seule condition que l'onde réfléchie par la cavité à la résonance soit en quadrature avec le vecteur de référence des bandes latérales [4]. On obtient ainsi un signal d'erreur qui, après amplification, est injecté sur l'élément de contrôle de la fréquence (varactor) de l'oscillateur à diode Gunn.

Dans la version modifiée par Stein et Turneaure [5], figure $1 b$, on effectue à la température ambiante, une modulation de phase à la pulsation $\Omega$ de l'onde délivrée par l'oscillateur. Cette onde modulée est envoyée dans la ligne de transmission alimentant la cavité de référence, de sorte que les fluctuations de phase dues aux fluctuations de longueur de ligne affectent simultanément et de la même manière l'onde porteuse et les bandes latérales de modulation. En toute rigueur, il faudrait tenir compte dans ce 


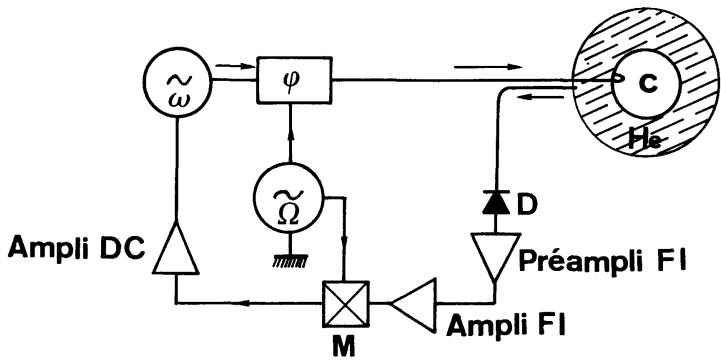

Fig. 1b. - Principe du discriminateur de Pound modifié.

[Block diagram of the modified IF Pound discriminator.]

dispositif, d'une fluctuation résiduelle de fréquence accompagnant les fluctuations de longueur, due à la dispersion de la ligne de transmission. Cette fluctuation résiduelle peut être négligée lorsque la fréquence de modulation est très petite devant $\omega$ $\left(\Omega / \omega=10^{-4}\right.$ dans notre cas), ou lorsqu'on utilise une alimentation par câbles coaxiaux au lieu de guides rectangulaires.

La détection de l'onde réfléchie prélevée par un coupleur directionnel fournit comme dans le cas précédent, un signal proportionnel à la partie imaginaire du coefficient de réflexion de la cavité. (On notera que les bandes latérales faisant partie de l'onde incidente se réfléchissent comme si la ligne était terminée par un court-circuit.)

2.2 LE DISPOSITIF DE MODULATION A LA FRÉQUENCE INTERMÉDIAIRE. - 2.2.1 Modulation interne. - Dans un souci de simplification, nous avons effectué une modulation interne qui consiste à superposer à la tension continue du varactor la tension délivrée par un oscillateur à quartz. L'indice de modulation est suffisamment faible pour que seules les deux premières bandes latérales soient conservées.

L'oscillateur est associé à une cavité supraconductrice en transmission qui joue ici le röle de filtre vis-à-vis des bandes latérales de modulation. Dans cette version, on utilise pratiquement toute la puissance disponible de l'oscillateur, aux pertes d'insertion près.

Nous avons, par ce procédé, obtenu un signal de très bonne pureté spectrale, pour une puissance utile de $10 \mathrm{~mW}$, mais la fréquence délivrée est fixe. Un accord de fréquence par variation de la fréquence de résonance de la cavité, à l'aide d'un dispositif mécanique, pose des problèmes difficiles à résoudre et entraîne une dégradation du coefficient de surtension.

2.2.2 Modulation externe : possibilité d'un accord électronique de fréquence. - Le principe de ce deuxième type de modulation, que nous avons mis au point, est représenté schématiquement sur la figure 2. L'onde délivrée par l'oscillateur se sépare en deux parties égales entre les voies (2) et (3) du té

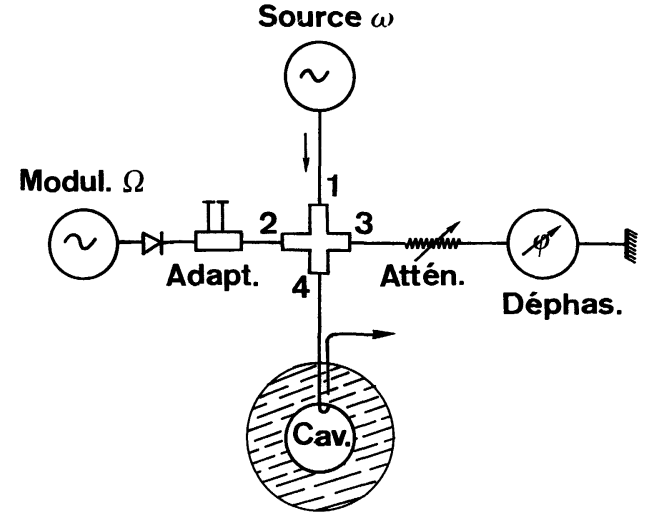

Fig. 2. - Principe du dispositif de modulation externe.

[Block diagram of the external modulation system.]

magique. La modulation est effectuée sur la voie (2), alors que la voie (3) est simplement terminée par un court-circuit. L'atténuateur variable placé sur cette voie permet de s'assurer à l'aide d'un analyseur de spectre que l'onde réfléchie par le cristal modulateur ne comprend que les bandes latérales de modulation. La suppression de l'onde porteuse y est effectuée grâce à l'adaptateur à pistons latéraux intercallé en aval du cristal modulateur. Le déphaseur placé dans la voie (3) permet alors d'ajuster à volonté le déphasage entre l'onde porteuse réfléchie par le court-circuit et le vecteur de référence des bandes latérales envoyées dans la voie (4) alimentant la

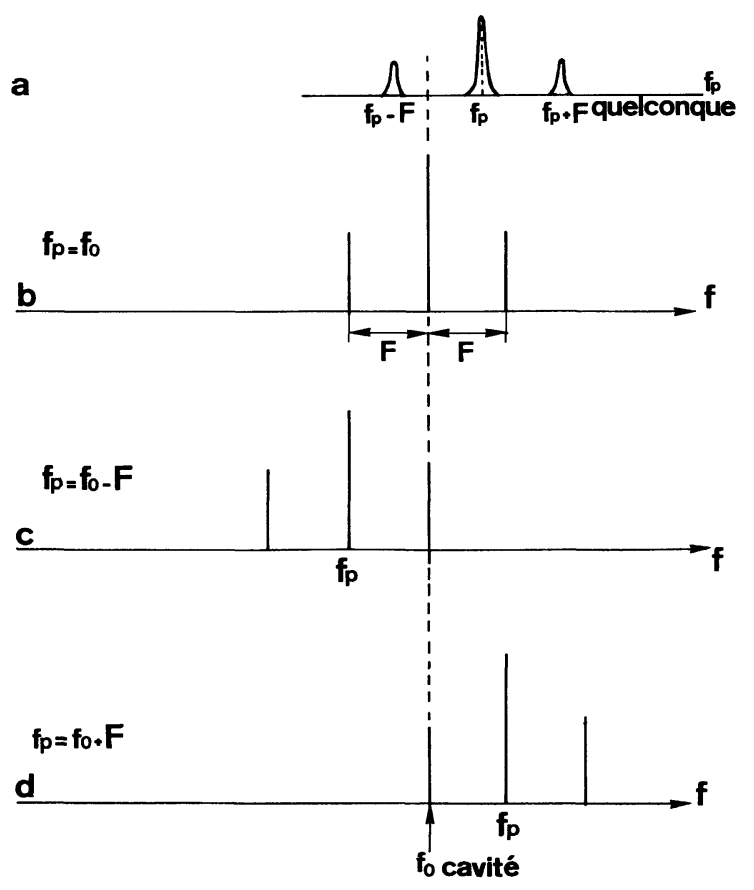

Fig. 3. - Représentation spectrale schématique de l'onde incidente en amont de la cavité stabilisatrice.

[Schematic spectral representation of the incident wave on the stabilizing cavity.] 
cavité. Suivant la valeur de ce déphasage, il est possible d'obtenir essentiellement deux modes de stabilisation que nous résumons par la représentation schématique de la figure 3 :

Si l'on désigne par $f_{0}$ la fréquence de résonance de la cavité, $f_{\mathrm{p}}$ la fréquence délivrée par l'oscillateur à diode Gunn et $F$ la fréquence intermédiaire de l'oscillateur auxiliaire à quartz, le cas (3a) correspond à une oscillation libre avec $f_{0}$ très différent à la fois de $f_{\mathrm{p}}$ et de $f_{\mathrm{p}} \pm F$.

Lorsque $f_{\mathrm{p}}=f_{0}$, on obtient la stabilisation classique qui correspond à une modulation en phase de l'onde (Fig. 3b).

Lorsque $f_{\mathrm{p}}=f_{0} \pm F$ (Figs. $3 c$ et $3 d$ ), on observe la stabilisation sur l'une des bandes latérales lorsque l'onde est modulée soit en phase, soit en amplitude, comme on peut le montrer à l'aide de la représentation graphique des figures 4 .

a)

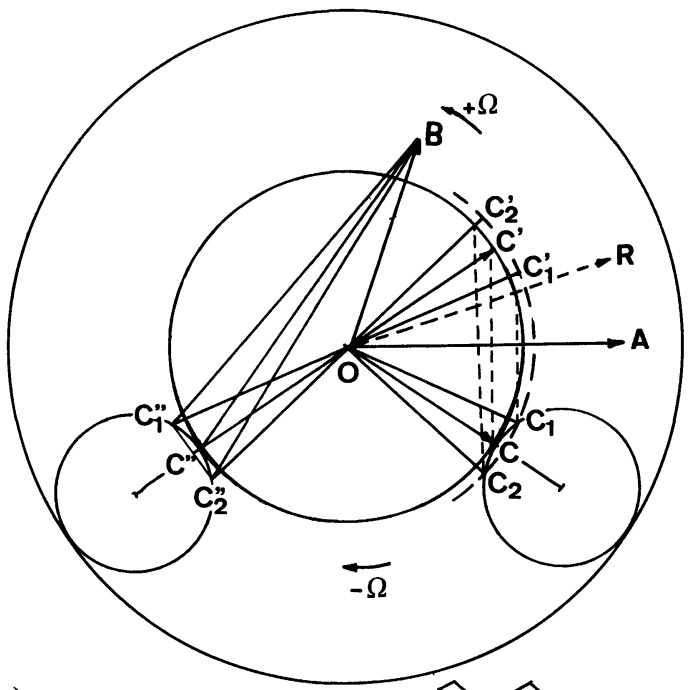

$\widehat{A O C}=\widehat{A O C} C^{\prime}=\Omega t+\varphi_{3}+\varphi_{1}$ $\widehat{\mathrm{AOB}}=\Omega \mathrm{t}+\varphi_{2}-\varphi_{1}$

b)

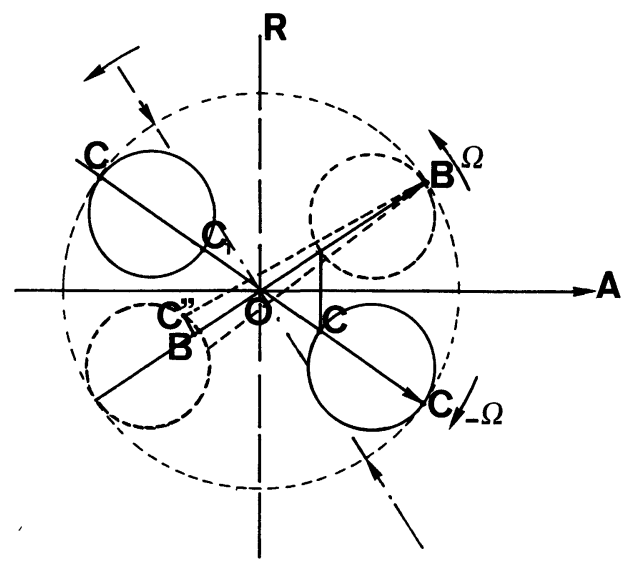

Fig. 4. - Représentation vectorielle du signal détecté à la fréquence intermédiaire. a) Cas général d'une onde incidente modulée en amplitude et en phase. b) Cas particuliers d'une onde incidente modulée en amplitude ou en phase.

[Vectorial representation of the IF detected signal. a) General case of an incident wave modulated both in amplitude and phase. b) Particular cases of an amplitude or phase-modulated incident wave.]
Considérons tout d'abord le cas général d'une onde réfléchie modulée à la fois en amplitude et en phase (Fig. 4a). L'onde UHF arrivant sur le cristal détecteur se compose de la porteuse $A \cos \left(\omega_{\mathrm{p}} t+\varphi_{1}\right)$ et les deux bandes latérales

$$
B \cos \left\{\left(\omega_{\mathrm{p}}+\Omega\right) t+\varphi_{2}\right\}+C \cos \left\{\left(\omega_{\mathrm{p}}-\Omega\right) t-\varphi_{3}\right\}
$$

On montre aisément [4] que la détection quadratique fournit un signal à fréquence intermédiaire de la forme :

$$
\begin{aligned}
V_{\mathrm{IF}}=R \cdot \alpha \cdot A\{B \cos (\Omega t & \left.+\varphi_{2}-\varphi_{1}\right)+ \\
& \left.+C \cos \left(\Omega t+\varphi_{3}+\varphi_{1}\right)\right\}
\end{aligned}
$$

où $R$ est l'impédance vidéo du cristal détecteur et $\alpha$ sa sensibilité en courant. Les termes entre crochets sont représentés par les vecteurs $\mathrm{OB}$ et $\mathrm{OC}^{\prime}$, symétrique de $\mathrm{OC}$ par rapport à $\mathrm{OA}$. $\mathrm{C}^{\prime \prime}$ étant le symétrique de $C^{\prime}$ par rapport à $O$, le vecteur $C^{\prime \prime} B$ représente la tension alternative à la sortie du cristal détecteur en amplitude et en phase. Si l'on suppose maintenant que la fréquence de l'une des bandes latérales coïncide avec la fréquence de résonance $f_{0}$ de la cavité, celle-ci se comportant comme un court-circuit vis-à-vis des deux autres composantes, un glissement de fréquence $\pm \delta f$ se traduira par un signal dissymétrique $\left(\mathrm{BC}_{1}^{\prime \prime} \neq \mathrm{BC}_{2}^{\prime \prime}\right)$ de part et d'autre de la résonance.

L'obtention d'une caractéristique de discrimination symétrique a lieu lorsque le vecteur de référence des bandes latérales est en phase (onde modulée en amplitude) ou en quadrature (onde modulée en phase) avec la porteuse (Fig. $4 b$ ). On notera que le signal détecté ne s'annule pas à la résonance, contrairement au cas classique $f_{\mathrm{p}}=f_{0}$. La proportionnalité à la partie imaginaire du coefficient de réflexion est obtenue après détection synchrone du signal délivré par le cristal détecteur, $V_{\mathrm{IF}}$, qui est injecté en quadrature de phase avec la tension de référence dans le mélangeur équilibré.

Dans le cas de la stabilisation de l'oscillateur sur une bande latérale $\left(f_{\mathrm{p}}=f_{0} \pm F\right)$, une variation de la fréquence intermédiaire $F$ entraînera la variation correspondante de la fréquence $f_{\mathrm{p}}$ du signal délivré par l'oscillateur Gunn, la stabilité en fréquence étant comparable dans les deux modes de stabilisation. En utilisant comme oscillateur local à la fréquence $F$ un synthétiseur piloté par quartz, nous avons pu faire varier continûment la fréquence de l'oscillateur stabilisé dans une plage de plusieurs $\mathrm{kHz}$, sans que la qualité du signal en soit affectée. Nous envisageons d'augmenter ultérieurement l'étendue de cette plage de variation, car elle dépend essentiellement de la bande passante des amplificateurs et filtres utilisés par le circuit de stabilisation. Il sera peut-être alors nécessaire de retoucher aux réglages des voies 2 et 3 (Fig. 2), afin de maintenir une phase et une adaptation correctes. 
3. Les différents éléments de la chaîne de contreréaction. - La détection quadratique est effectuée à l'aide d'une diode tunnel ayant une sensibilité de $200 \mathrm{mV} / \mathrm{mW}$. Cette diode tunnel est suivie d'un préamplificateur et amplificateur ayant un gain global de $65 \mathrm{~dB}$ et une bande passante de $1 \mathrm{MHz}$. Les bandes latérales d'ordre supérieur sont supprimées par des filtres de réjection centrés à $2 \mathrm{MHz}$. Le mélangeur équilibré présente une bande passante de 0,2 à $500 \mathrm{MHz}$.

L'amplificateur continu ayant un gain de $50 \mathrm{~dB}$ est constitué de trois filtres actifs du $2^{e}$ ordre. Les zéros et pôles successifs ont été déterminés de manière à obtenir une pente moyenne de gain de $30 \mathrm{~dB}$ par décade et un déphasage d'environ $90^{\circ}$ à partir de la fréquence $f_{R}=f_{0} / 2 Q_{\mathrm{L}}$, inverse du temps de remplissage $\tau_{\mathrm{r}}$ de la cavité qui joue le rôle d'un filtre passebas dans la boucle de contre-réaction ( $f_{0}$ est la fréquence de résonance de la cavité et $Q_{\mathrm{L}}$ son coefficient de surtension en charge). Le gain total en boucle ouverte est de la forme :

$$
G_{\mathrm{T}}=\left(\frac{f_{1}}{f}\right)^{3 / 2}
$$

$f_{1}$ étant la fréquence de coupure de la boucle.

En ce qui concerne le bruit thermique introduit par le dispositif électronique, on montre aisément [6] qu'il peut s'exprimer sous la forme :

$$
S_{\mathrm{y}}(f)=4 k T\left(R+R_{\mathrm{A}}\right)\left\{8 \alpha R P_{\mathrm{c}} Q_{\mathrm{L}} \frac{B}{A} \frac{2 \beta}{1+\beta}\right\}^{-2}
$$

où $S_{\mathrm{y}}(f)$ représente la densité spectrale des fluctuations relatives de fréquence, $\boldsymbol{R}_{\mathrm{A}}$ l'impédance d'entrée du préamplificateur, $R$ l'impédance vidéo du cristal détecteur et $\alpha$ sa sensibilité, $P_{\mathrm{c}}$ la puissance injectée dans la cavité, $B / A$ l'indice de modulation, $\beta$ le coefficient de couplage à la ligne de transmission et $T$ la température.

En tenant compte de la valeur de ces paramètres, on obtient $S_{\mathrm{y}}(f) \simeq 10^{-28} \mathrm{~Hz}^{-1}$, qui montre que le bruit thermique pourra, en général, être négligé.

4. Caractérisation de l'oscillateur : stabilité, pureté spectrale. - Les propriétés de l'oscillateur à diode Gunn stabilisé par une cavité supraconductrice ont été étudiées selon les méthodes conventionnelles [7, 8] dans le domaine temps, puis dans le domaine fréquence. Dans le domaine temps, la caractérisation a été effectuée au moyen du calcul de la variance d'Allan à deux échantillons :

$$
\sigma_{\mathrm{y}}^{2}\left(2,2 N, \tau, \tau_{\mathrm{m}}\right)=\frac{1}{f_{\mathrm{p}}^{2}}\left[\frac{\sum_{i=1}^{N}\left(f_{2 i n}-f_{2 i}\right)^{2}}{2 N}\right]
$$

où $f_{\mathrm{p}}$ est la fréquence moyenne de l'oscillateur, $N$ le nombre de couples de mesures de fréquence faite chacune pendant le temps $\tau$ et séparés par un temps mort $\tau_{\mathrm{m}}$. Nous avons choisi $2 N=100$; $\tau$ est fixé à volonté dans le domaine $(1 \mathrm{~ms}-10 \mathrm{~s})$ et le temps mort inévitable est d'environ $1 \mathrm{~ms}$.

Nos premières mesures portant sur les stabilités obtenues lorsque l'oscillateur est asservi, d'une part à la fréquence de résonance de la cavité $\left(f_{\mathrm{p}}=f_{0}\right)$, d'autre part lorsque sa fréquence est décalée de la fréquence in termédiaire $F\left(f_{\mathrm{p}}=f_{0} \pm F\right)$, ont montré que dans les deux cas, on obtient une loi de variation de $\sigma_{y}$ du type $\sigma_{y} \simeq 3 \times 10^{-12} \tau^{-1}$ pour $1 \mathrm{~ms} \leqslant \tau \leqslant 1 \mathrm{~s}$. Ces valeurs correspondent en fait à la limite de résolution du compteur-calculateur HP 5360 A utilisé [9]. Pour s'affranchir de cette limitation, nous avons effectué un battement entre deux oscillateurs à diode Gunn identiques, stabilisés chacun par une cavité supraconductrice de fréquences de résonance $f_{0}$ et $f_{0}^{\prime}$. Selon l'accord électronique de chacun des oscillateurs stabilisés, on obtient les fréquences de battement $\left(f_{0}-f_{0}^{\prime}\right) ;\left(f_{0}-f_{0}^{\prime} \pm F\right) ;\left(f_{0}-f_{0}^{\prime} \pm 2 F\right)$. Le calcul de la variance d'Allan dans ces différents cas conduit au même résultat qui est reporté sur la courbe (a) de la figure 5 :

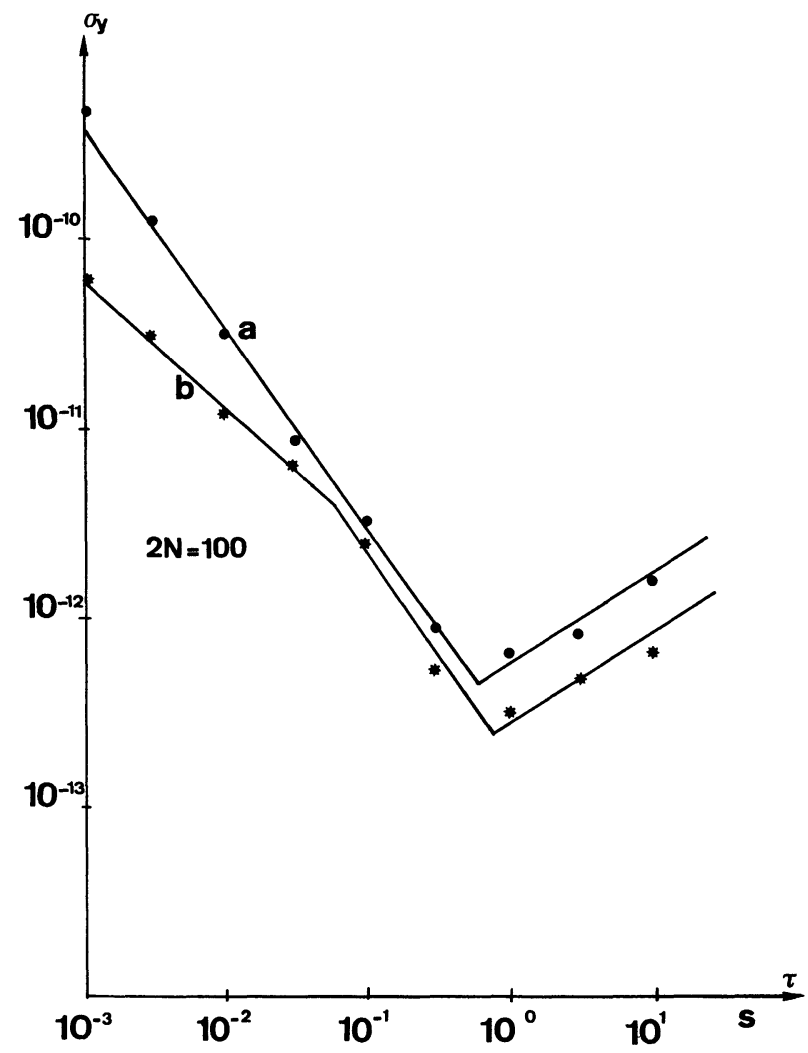

Fig. 5. - Variance d'Allan à deux échantillons des fluctuations relatives de fréquence en fonction du temps de comptage $\sigma_{y}(\tau)$. a) Battement des ondes délivrées par les deux oscillateurs à diode Gunn asservis par deux cavités supraconductrices. b) Battement des ondes transmises par les deux cavités stabilisatrices.

[Two sample Allan variance of the fractional frequency fluctuations versus averaging time $\sigma_{y}(\tau)$. a) Beat frequency between two superconducting-cavity-stabilized Gunn effect oscillators. $b$ ) Beat frequency between the waves transmitted by the two stabilizing cavities.] 
- pour les valeurs $\tau<1 \mathrm{~s}$ environ, la loi de variation est du type $\sigma_{\mathrm{y}}(\tau) \simeq 2 \times 10^{-13} \tau^{-1}$, pour une bande passante de mesure de $100 \mathrm{kHz}$.

- Pour $\tau>1 \mathrm{~s}$, on observe une croissance lente de $\sigma_{\mathrm{y}}$ que l'on peut attribuer à un bruit du type marche aléatoire de fréquence (loi en $\tau^{+1 / 2}$ ).

L'influence du filtrage du bruit par la cavité supraconductrice a été mise en évidence en mesurant la variance du signal de battement à la fréquence $\left(f_{0}-f_{0}^{\prime}\right)$ entre les signaux transmis par chacune des cavités de référence utilisées (courbe b de la figure 5). Le résultat obtenu est le suivant :

- Pour $\tau<\tau_{\mathrm{r}}$, la loi de variation de $\sigma_{\mathrm{y}}$ est sensiblement en $\tau^{-1 / 2}$ : le bruit blanc de phase est transformé en bruit blanc de fréquence.

$\begin{array}{cccc}f(\mathrm{~Hz}) & 15 & 50 & 100 \\ S_{\varphi}\left(\mathrm{rad}^{2} / \mathrm{Hz}\right) & 1,7 \times 10^{-8} & 1,2 \times 10^{-8} & 5,8 \times 10^{-8}\end{array}$

Cette mesure fait apparaître une région spectrale $\left(f<f_{R} \simeq 150 \mathrm{~Hz}\right)$ de bruit blanc de phase d'un niveau de quelque $10^{-8} \mathrm{rad}^{2} / \mathrm{Hz}$ et une autre $\left(f_{R}<f<1500 \mathrm{~Hz}\right)$ caractérisée par une décroissance d'environ $20 \mathrm{~dB}$ par décade qui peut être identifiée comme étant du bruit blanc de fréquence. Dans notre cas, la relation théorique entre $S_{\varphi}(f)$ et $\sigma_{\mathrm{y}}(\tau)$ par un bruit blanc de phase s'écrit (filtre à pôle simple de fréquence de coupure $f_{\mathrm{c}}$ ) :

$$
\sigma_{y}^{2}(\tau)=\frac{3 f_{\mathrm{c}}}{8 \pi v^{2} \tau^{2}} S_{\varphi}(f)
$$

Pour $S_{\varphi}(f)=1,5 \times 10^{-8} \mathrm{rad}^{2} / \mathrm{Hz}, f_{0}=f_{R}=150 \mathrm{~Hz}$, $v=10^{10} \mathrm{~Hz}$, on obtient :

$$
\sigma_{y}(\tau) \simeq 5,2 \times 10^{-14} \tau^{-1} .
$$

A titre de comparaison, les performances que nous avons mesurées dans le domaine fréquence sont du même ordre que les prévisions les plus optimistes que l'on peut faire à l'heure actuelle en partant d'un quartz de $5 \mathrm{MHz}$ à faible bruit du type Austron $1120 \mathrm{~S}$ après une multiplication de fréquence idéale. Mais dans la réalité, la chaîne de multiplication ellemême introduit un bruit de phase supplémentaire [10].
- Pour $\tau_{\mathrm{r}}<\tau<10 \mathrm{~s}$, on retrouve les variations en $\tau^{-1}$ et en $\tau^{+1 / 2}$, analogues à celle du montage précédent.

La caractérisation dans le domaine fréquence a été effectuée en réalisant un second battement à fréquence nulle, par utilisation d'un synthétiseur de fréquence auxiliaire. Cette analyse a montré la présence de raies discrètes à basse fréquence $(f<150 \mathrm{~Hz})$ dues à des vibrations mécaniques que l'on s'est efforcé de réduire en utilisant des vérins pneumatiques pour supporter le cryostat.

Le tableau ci-après montre le résultat des mesures de la densité spectrale des fluctuations de phase $S_{\varphi}(f)$ pour quelques points du spectre de Fourier, dans le cas de signaux prélevés sur la voie de transmission des cavités de référence.

$\begin{array}{ccccc}150 & 200 & 800 & 1000 & 1500 \\ 10^{-8} & 3 \times 10^{-9} & .1,2 \times 10^{-10} & 1,7 \times 10^{-10} & 8 \times 10^{-11}\end{array}$

5. Conclusion. - En mettant à profit les qualités remarquables des cavités supraconductrices en UHF, nous avons mis au point un oscillateur à diode Gunn de haute pureté spectrale et qu'il est possible d'accorder électroniquement à l'aide d'un synthétiseur de fréquence. Cet oscillateur sera utilisé prochainement comme sources de base dans une expérience de multiplication de fréquence par jonction Josephson. L'objectif à plus long terme est la métrologie des fréquences des lasers dans l'infrarouge lointain (raies $118 \mu \mathrm{m}$ et $70 \mu \mathrm{m}$ du laser à alcool méthylique). D'autres applications peuvent dès maintenant être envisagées dans le domaine de la métrologie, comme par exemple la détermination précise des niveaux de Rydberg du Na [11].

Remerciements. - Les auteurs tiennent à remercier tout particulièrement le Dr J. P. Turneaure et le Dr S.R. Stein du HEPL de l'Université de Stanford qui leur ont fourni des renseignements très précieux pour la réussite de ce travail, ainsi que le Dr H. A. Schwettman qui a bien voulu mettre à leur disposition les diverses installations de son laboratoire pour la mise au point des cavités.

Ils remercient également MM. Thély et Guével de l'ENSET de Cachan qui ont bien voulu se charger de l'usinage délicat des cavités.

\section{Bibliographie}

[1] Turneaure, J. P., Nguyen Tuong Viet, Appl. Phys. Lett. $16(1970) 333$.

[2] HAlBRITTER, J., Externer Bericht 3/68-8 (Kernforschgszentrum, Karlsruhe) (1968)

[3] Trela, W. J., Thèse, Université de Stanford (1966) pp. 36-41.

[4] Muller, F., Thèse de Doctorat de $3^{\mathrm{e}}$ cycle, Orsay (1964).

[5] Stein, S. R., Turneaure, J.-P., Proc. 27th An. Symp. on Frequency Control (Elec. Ind. Assoc., Washington DC, 1973), pp. 414-420.
[6] Stein, R. S., Thèse Ph. D., Université de Stanford (1974).

[7] HowE, D. A., Frequency domain stability measurements. A tutorial introduction, NBS Technical Note 679 (1976).

[8] Audorn, C., Frequency Metrology, Cours de l'Ecole d'Eté Enrico Fermi, juillet 1976, Varenne (Italie).

[9] JimeneZ, J. J., Thèse de Doctorat d'Etat, Orsay (1974).

[10] Bava, E., De Marchi, A., Godone, A., Proc. 31 An. Symp. on Frequency Control, Fort Monmouth (1977).

[11] Goy, P., Communication privée. 